\title{
FINITENESS PROPERTIES GENERALIZED LOCAL COHOMOLOGY WITH RESPECT TO AN IDEAL CONTAINING THE IRRELEVANT IDEAL
}

\author{
FAtemeh Dehghani-Zadeh
}

\begin{abstract}
The membership of the generalized local cohomology modules $H_{\mathfrak{a}}^{i}(M, N)$ of two $R$-modules $M$ and $N$ with respect to an ideal $\mathfrak{a}$ in certain Serre subcategories of the category of modules is studied from below $(i<t)$. Furthermore, the behaviour of the $n$th graded component $H_{\mathfrak{a}}^{i}(M, N)_{n}$ of the generalized local cohomology modules with respect to an ideal containing the irrelevant ideal as $n \rightarrow-\infty$ is investigated by using the above result, in certain graded situations.
\end{abstract}

\section{Introduction}

Generalized local cohomology was given in the local case by J. Herzog [7] and in the more general case by Bijan-Zadeh [1]. Let $R$ be a commutative Noetherian ring with identity, $\mathfrak{a}$ an ideal of $R$ and let $M, N$ be two $R$-modules. For an integer $i \geq 0$, the $i$ th generalized local cohomology module $H_{\mathfrak{a}}^{i}(M, N)$ is defined by $H_{\mathfrak{a}}^{i}(M, N)=\lim _{n \in \mathbb{N}} \operatorname{Ext}_{R}^{i}\left(M / \mathfrak{a}^{n} M, N\right)$. With $M=R$, we obtain the ordinary local cohomology module $H_{\mathfrak{a}}^{i}(N)$ of $N$ with respect to a which was introduced by Grothendieck.

Recall that a class $S$ of $R$-modules is a Serre subcategory of the category of $R$-modules, when it is closed under taking submodules, quotients and extensions. In this paper, we study some properties of generalized local cohomology modules by using Serre classes.

This paper is divided into three sections. In the second section of the paper, we show that if $M$ is a finitely generated $R$-module, $N$ is an arbitrary $R$-module and $t$ is a non-negative integer such that $H_{\mathfrak{a}}^{i}(N) \in S$ for all $i<t$, then

(i) $H_{\mathfrak{a}}^{i}(M, N)$ is in $S$ for all $i<t$ (Theorem 2.1),

(ii) $\operatorname{Ext}_{R}^{j}\left(\frac{R}{\mathfrak{a}}, H_{\mathfrak{a}}^{i}(N)\right)$ is in $S$ for $j=0,1$ and $i \leq t$, where $N$ is an $R$-module in $S$ (Theorem 2.2),

Received April 29, 2011; Revised April 23, 2012.

2010 Mathematics Subject Classification. 13D45, 13D07.

Key words and phrases. generalized local cohomology, finiteness, Serre subcategories. 
(iii) $\operatorname{Hom}\left(R / \mathfrak{a}, H_{\mathfrak{a}}^{i}(M, N)\right)$ is in $S$ for all $i \leq t$, where $N$ is an $R$-module in $S$ (Theorem 2.4).

Throughout Section $3, R=\bigoplus_{n \geq 0} R_{n}$ is a graded commutative Noetherian ring, where the base ring $R_{0}$ is a commutative Noetherian local ring with maximal ideal $\mathfrak{m}_{0}$. Moreover, we use $\mathfrak{a}_{0}$ to denote a proper ideal of $R_{0}$ and we set $R_{+}=\bigoplus_{n>0} R_{n}$ the irrelevant ideal of $R, \mathfrak{a}=\mathfrak{a}_{0}+R_{+}$, and $\mathfrak{m}=$ $\mathfrak{m}_{0}+R_{+}$. Also, we use $M=\bigoplus_{n \in \mathbb{Z}} M_{n}$ and $N=\bigoplus_{n \in \mathbb{Z}} N_{n}$ to denote non-zero, finitely generated graded $R$-modules. It is well known that, the $i$ th generalized local cohomology module $H_{\mathfrak{a}}^{i}(M, N)$ inherits natural grading for each $i \in \mathbb{N}_{0}$ (where $\mathbb{N}_{0}$ denotes the set of all non-negative integers). We use the notation $H_{\mathfrak{a}}^{i}(M, N)_{n}$ to denote the $n$th graded component of $H_{\mathfrak{a}}^{i}(M, N)$ for each $n \in \mathbb{Z}$ (where $\mathbb{Z}$ denotes the set of integers). Then, according to [8], for each $i \geq 0$, the $R_{0}$-module $H_{\mathfrak{a}}^{i}(M, N)_{n}$ is finitely generated in certain cases and vanishes for all $n \gg 0$. The cohomological dimension of $M$ and $N$ with respect to $\mathfrak{a}$ is denoted by $\operatorname{cd}_{\mathfrak{a}}(M, N)$. Thus $\operatorname{cd}_{\mathfrak{a}}(M, N)$ is the largest non-negative integer $i$ such that $H_{\mathfrak{a}}^{i}(M, N)$ is not equal to zero. The finiteness dimension of $M$ and $N$ with respect to $\mathfrak{a}$ is denoted by $f_{\mathfrak{a}}(M, N)$, where $f_{\mathfrak{a}}(M, N)$ is the least non-negative integer $i$ such that $H_{\mathfrak{a}}^{i}(M, N)$ is not finitely generated. Also, following [5], the generalized homological finite length dimension of $N$ and $M$ is defined with respect to $\mathfrak{a}$ as

$$
g_{\mathfrak{a}}(M, N)=\inf \left\{i \in \mathbb{N}_{0} \mid l_{R_{0}} H_{\mathfrak{a}}^{i}(M, N)_{n}=\infty \text { for infinitely many } n \in \mathbb{Z}\right\},
$$

where we denote by $l_{R_{0}} T$ the length over $R_{0}$ of $T$ for an $R_{0}$-module $T$.

In Section 3, using the above results, we study the asymptotic behaviour of $R_{0}$ module $H_{\mathfrak{a}}^{i}(M, N)_{n}$ as $n$ tends to $-\infty$, in the following cases;

(i) $\left(R_{0}, \mathfrak{m}_{0}\right)$ is local and $\operatorname{dim} R_{0} / \mathfrak{a}_{0}=1$ (Theorem 3.13),

(ii) $i \leq g_{\mathfrak{a}}(M, N)=g$ and $\Gamma_{\mathfrak{a}_{0}}(M)=M$ (Theorem 3.10),

(iii) $i=d+\ell$, where $\operatorname{pd}(M)=\ell$, the projective dimension of $M$, and $\operatorname{dim} N / \mathfrak{b}_{0} N=d$ (Lemma 3.8).

This work is motivated by article of Jahangiri and Zakeri [8]. While editing this paper, we realized that Theorem 3.10 is proven with the assumption that the projective dimension of $M$ is finite [9]. In this paper the result is proved, without imposing the assumption finiteness of projective dimension of $M$, with different arguments.

For any unexplained terminology, the reader can refer to [2], [3] and [10].

\section{Serre classes}

This section is started with the following theorem.

Theorem 2.1. Let $\mathfrak{a}$ be an ideal of $R$ and $M$ a finitely generated $R$-module and $N$ an arbitrary $R$-module. Let $r$ be an integer such that $H_{\mathfrak{a}}^{i}(N)$ is in $S$ for all $i<r$. Then $H_{\mathfrak{a}}^{i}(M, N)$ is in $S$ for all $i<r$. 
Proof. Consider the function $F(-)=\operatorname{Hom}(M,-)$ and $G(-)=\Gamma_{\mathfrak{a}}(-)$. Then one has $F(G(-))=\operatorname{Hom}\left(M, \Gamma_{\mathfrak{a}}(-)\right)=H_{\mathfrak{a}}^{0}(M,-)$. Let $E$ be an injective $R$ module. Then it is known that $H_{a}^{0}(E)$ is also an injective $R$-module. Hence $\operatorname{Ext}_{R}^{i}\left(M, \Gamma_{\mathfrak{a}}(E)\right)=0$ for all $i>0$. So, by [14, 11.38], there is a Grothendieck's spectral sequence

$$
E_{2}^{p, q}=\operatorname{Ext}_{R}^{p}\left(M, H_{\mathfrak{a}}^{q}(N)\right) \Longrightarrow H_{\mathfrak{a}}^{p+q}(M, N) .
$$

Since the modules $H_{\mathfrak{a}}^{0}(N), H_{\mathfrak{a}}^{1}(N), \ldots, H_{\mathfrak{a}}^{r-1}(N)$ are in $S$, and $M$ is a finitely generated $R$-module hence $E_{2}^{p . q}$ is in $S$ for all $p \geq 0$ and $q<r$, see [6,2.1]. For all $i \geq 2, t<r$ and $p \geq 0$, we consider the exact sequence

$$
0 \longrightarrow \operatorname{kerd}_{i}^{p, t} \longrightarrow E_{i}^{p, t} \longrightarrow E_{i}^{p+i, t-i+1} .
$$

Since $E_{i}^{p, t}=\frac{\operatorname{ker} d_{i-1}^{p, t}}{\operatorname{im} d_{i-1}^{p-i+1, t+i-2}}$ and $E_{i}^{p, j}=0$ for all $j<0$, we use $(*)$ to obtain $\operatorname{kerd}_{t+2}^{i, t-i} \cong E_{t+2}^{i, t-i} \cong \ldots \cong E_{\infty}^{i, t-i}$ for all $0 \leq i \leq t$. There exists a finite filtration

$$
0=\varphi^{t+1} H^{t} \subseteq \varphi^{t} H^{t} \subseteq \cdots \subseteq \varphi^{1} H^{t} \subseteq \varphi^{0} H^{t}=H_{\mathfrak{a}}^{t}(M, N)
$$

such that $E_{\infty}^{i, t-i}=\frac{\varphi^{i} H^{t}}{\varphi^{i+1} H^{t}}$ for all $0 \leq i \leq t$.

Now, the exact sequence

$$
0 \longrightarrow \varphi^{i+1} H^{t} \longrightarrow \varphi^{i} H^{t} \longrightarrow E_{\infty}^{i, t-i} \longrightarrow 0 \quad(0 \leq i \leq t)
$$

in conjunction with $E_{\infty}^{i, t-i} \cong \operatorname{ker} d_{t+2}^{i, t-i} \subseteq \operatorname{ker} d_{2}^{i, t-i} \subseteq E_{2}^{i, t-i}$ yields $H_{\mathfrak{a}}^{i}(M, N)$ is in $S$ for all $0 \leq i<r$.

Theorem 2.2. Let $\mathfrak{a}$ be an ideal of $R$ and $N$ an $R$-module in $S$. Let $t$ be an integer such that $H_{\mathfrak{a}}^{i}(N)$ is in $S$ for all $i<t$. Then $\operatorname{Ext}_{R}^{j}\left(R / \mathfrak{a}, H_{\mathfrak{a}}^{i}(N)\right)$ is in $S$ for all $i \leq t$ and $j=0,1$.

Proof. Using $[14,11.38]$ there exists a Grothendieck's spectral sequence

$$
E_{2}^{p, q}=\operatorname{Ext}_{R}^{p}\left(R / \mathfrak{a}, H_{\mathfrak{a}}^{q}(N)\right) \Longrightarrow \operatorname{Ext}_{R}^{p+q}(R / \mathfrak{a}, N) .
$$

Also, there is a bounded filtration

$$
0=\varphi^{t+1} H^{t} \subseteq \varphi^{t} H^{t} \subseteq \cdots \subseteq \varphi^{1} H^{t} \subseteq \varphi^{0} H^{t}=\operatorname{Ext}_{R}^{t}(R / \mathfrak{a}, N)
$$

such that $E_{\infty}^{i, t-i} \cong \frac{\varphi^{i} H^{t}}{\varphi^{i+1} H^{t}}$ for all $0 \leq i \leq t$. $\operatorname{Ext}_{R}^{i}(R / \mathfrak{a}, N)$ is in $S$ for all $i$, by $[6,2.1]$, and hence $E_{\infty}^{p, q}$ is in $S$ for all $p, q$. Note that $E_{\infty}^{p, q}=E_{r}^{p, q}$ for large $r$ and each $p$ and $q$. It follows that there is an integer $\ell \geq 2$ such that $E_{r}^{p, q}$ is in $S$ for all $r \geq \ell$. We now argue by descending induction on $\ell$. Now, assume that $2<\ell<r$ and that the claim holds for $\ell$. Since $E_{r}^{p, q}$ is in a subquotient of $E_{2}^{p, q}$ for all $p, q \in \mathbb{N}_{0}$, the hypotheses in conjunction with $[6,2.1]$ give $E_{r}^{p+r, t-r+1}$ is in $S$ for all $r \geq 2$. In addition, $E_{\ell}^{p, t}=\frac{\operatorname{ker} d_{\ell-1}^{p, t}}{\operatorname{im} d_{\ell-1}^{p-\ell+1, t+\ell-2}}$ and $\operatorname{im} d_{\ell-1}^{p-\ell+1, t+\ell-2}=0$ 
for $p=0,1$, it follows that $\operatorname{ker} d_{\ell-1}^{p, t}$ is in $S$ for all $\ell>2$ and $p=0,1$. Let $r \geq 2$ and $p=0,1$, we consider the sequence

$$
0 \longrightarrow \operatorname{kerd}_{r}^{p, t} \longrightarrow E_{r}^{p, t} \longrightarrow E_{r}^{p+r, t-r+1} .
$$

Since both $\operatorname{ker} d_{\ell-1}^{p, t}$ and $E_{\ell-1}^{p+r, t-r+1}$ are in $S$, it follows that $E_{\ell-1}^{p, t}$ is in $S$ for $p=0,1$. This completes the inductive step.

Notation. For any $R$-module $T$, we denote by $\operatorname{pd}(T)$ the projective dimension over $R$ of $T$.

Corollary 2.3. Let $\mathfrak{a}$ be an ideal of $R, N$ an $R$-module in $S$ and $M$ a finitely generated $R$-module with $p d(M)<\infty$. Let $t$ be an integer such that $H_{\mathfrak{a}}^{i}(N)$ is in $S$ for all $i<t$. Then $\operatorname{Hom}\left(R / \mathfrak{a}, H_{\mathfrak{a}}^{i}(M, N)\right)$ is in $S$ for all $i \leq t$.

Proof. In view of Theorem $2.1 H_{\mathfrak{a}}^{i}(M, N)$ is in $S$ for all $i<t$. Hence

$$
\operatorname{Hom}\left(R / \mathfrak{a}, H_{\mathfrak{a}}^{i}(M, N)\right)
$$

is in $S$ for all $i<t$, by [6, 2.1]. It is enough for us to show that

$$
\operatorname{Hom}\left(R / \mathfrak{a}, H_{\mathfrak{a}}^{t}(M, N)\right)
$$

is in $S$. We prove the result by induction on $\operatorname{pd}(M)=\ell$. Using Theorem 2.2, the assertion holds for $\ell=0$. Now assume that $\ell>0$ and consider the exact sequence

$$
0 \longrightarrow M^{\prime} \longrightarrow R^{n} \longrightarrow M \longrightarrow 0 .
$$

It follows that $\operatorname{pd}\left(M^{\prime}\right) \leq \ell-1$. This short exact sequence yields the exact sequence

$$
\begin{aligned}
H_{\mathfrak{a}}^{t-1}\left(R^{n}, N\right) & \stackrel{\theta}{\longrightarrow} H_{\mathfrak{a}}^{t-1}\left(M^{\prime}, N\right) \stackrel{\varphi}{\longrightarrow} H_{\mathfrak{a}}^{t}(M, N) \\
& \stackrel{\psi}{\longrightarrow} H_{\mathfrak{a}}^{t}\left(R^{n}, N\right) \stackrel{\lambda}{\longrightarrow} H_{\mathfrak{a}}^{t}\left(M^{\prime}, N\right) \longrightarrow H_{\mathfrak{a}}^{t+1}(M, N) .
\end{aligned}
$$

If we break this sequence in three exact sequences

$$
\begin{gathered}
0 \longrightarrow \operatorname{im} \theta \longrightarrow H_{\mathfrak{a}}^{t-1}\left(M^{\prime}, N\right) \longrightarrow \operatorname{im} \varphi \longrightarrow 0, \\
0 \longrightarrow \operatorname{im} \varphi \longrightarrow H_{\mathfrak{a}}^{t}(M, N) \longrightarrow \operatorname{im} \psi \longrightarrow 0, \\
0 \longrightarrow \operatorname{im} \psi \longrightarrow H_{\mathfrak{a}}^{t}\left(R^{n}, N\right) \longrightarrow \operatorname{im} \lambda \longrightarrow 0,
\end{gathered}
$$

then we get the following exact sequences

(1) $\operatorname{Hom}\left(R / \mathfrak{a}, H_{\mathfrak{a}}^{t-1}\left(M^{\prime}, N\right) \longrightarrow \operatorname{Hom}(R / \mathfrak{a}, \operatorname{im} \varphi) \longrightarrow \operatorname{Ext}_{R}^{1}(R / \mathfrak{a}, \operatorname{im} \theta)\right.$,

(2) $0 \longrightarrow \operatorname{Hom}(R / \mathfrak{a}, \operatorname{im} \varphi) \longrightarrow \operatorname{Hom}\left(R / \mathfrak{a}, H_{\mathfrak{a}}^{t}(M, N)\right) \longrightarrow \operatorname{Hom}(R / \mathfrak{a}, \operatorname{im} \psi)$,

(3) $0 \longrightarrow \operatorname{Hom}(R / \mathfrak{a}, \operatorname{im} \psi) \longrightarrow \operatorname{Hom}\left(R / \mathfrak{a}, H_{\mathfrak{a}}^{t}\left(R^{n}, N\right)\right) \longrightarrow \operatorname{Hom}(R / \mathfrak{a}, \operatorname{im} \lambda)$.

By using the inductive hypothesis the $R$-module $\operatorname{Hom}\left(R / \mathfrak{a}, H_{\mathfrak{a}}^{t-1}\left(M^{\prime}, N\right)\right)$ is in $S$. Also, in view of $[6,2.1], \operatorname{Ext}_{R}^{1}(R / \mathfrak{a}, \operatorname{im} \theta)$ is in $S$. Therefore, according to the exact sequence $(1), \operatorname{Hom}(R / \mathfrak{a}, \operatorname{im} \varphi)$ is in $S$. On the other hand the last displayed exact sequence and Theorem 2.2, it follows that $\operatorname{Hom}(R / \mathfrak{a}, \operatorname{im} \psi)$ is in $S$. Now, we can use the exact sequence (2) to obtain the result. 
Theorem 2.4. Let $\mathfrak{a}$ be an ideal of $R, N$ an $R$-module in $S$ and $M$ a finitely generated $R$-module. Let $t$ be an integer such that $H_{\mathfrak{a}}^{i}(N)$ is in $S$ for all $i<t$. Then $\operatorname{Hom}\left(R / \mathfrak{a}, H_{\mathfrak{a}}^{i}(M, N)\right)$ is in $S$ for all $i \leq t$

Proof. We prove the theorem by induction on $t$. It is straightforward to see that the result is true when $t=0$. Suppose that $0<t$ and that the result has been proved for $t-1$. Now, we can use the exact sequence $0 \longrightarrow \Gamma_{\mathfrak{a}}(N) \longrightarrow$ $N \longrightarrow N / \Gamma_{\mathfrak{a}}(N) \longrightarrow 0$, in conjunction with the fact that $N$ is in $S$, to see that $H_{\mathfrak{a}}^{i}(M, N)$ is in $S$ if and only if $H_{\mathfrak{a}}^{i}\left(M, N / \Gamma_{\mathfrak{a}}(N)\right)$ is in $S$, also this holds for $H_{\mathfrak{a}}^{i}(N)$. We may assume that $\Gamma_{\mathfrak{a}}(N)=0$. Then there exists $x \in \mathfrak{a}$ such that $x$ is an $N$-sequence. Now, we may consider the exact sequence

$$
0 \longrightarrow N \stackrel{x}{\longrightarrow} N \longrightarrow N / x N \longrightarrow 0
$$

to obtain the exact sequences

$$
\begin{gathered}
H_{\mathfrak{a}}^{t-1}(N) \longrightarrow H_{\mathfrak{a}}^{t-1}(N / x N) \longrightarrow H_{\mathfrak{a}}^{t}(N) \stackrel{x}{\longrightarrow} H_{\mathfrak{a}}^{t}(N), \\
H_{\mathfrak{a}}^{t-1}(M, N) \stackrel{\lambda}{\longrightarrow} H_{\mathfrak{a}}^{t-1}(M, N / x N) \stackrel{\varphi}{\longrightarrow} H_{\mathfrak{a}}^{t}(M, N) \stackrel{x}{\longrightarrow} H_{\mathfrak{a}}^{t}(M, N) .
\end{gathered}
$$

Now, one can use the above exact sequences in conjunction with the inductive hypothesis and Theorem 2.1 to see that the $R$-module

$$
\operatorname{Hom}\left(R / \mathfrak{a}, H_{\mathfrak{a}}^{t-1}(M, N / x N)\right)
$$

is in $S$. Using the exact sequence

$$
\begin{aligned}
0 \longrightarrow \operatorname{Hom}(R / \mathfrak{a}, \operatorname{im} \lambda) & \longrightarrow \operatorname{Hom}\left(R / \mathfrak{a}, H_{\mathfrak{a}}^{t-1}(M, N / x N)\right. \\
& \longrightarrow \operatorname{Hom}(R / \mathfrak{a}, \operatorname{im} \varphi) \longrightarrow \operatorname{Ext}_{R}^{1}(R / \mathfrak{a}, \operatorname{im} \lambda)
\end{aligned}
$$

shows that

$$
\operatorname{Hom}(R / \mathfrak{a}, \operatorname{im} \varphi) \cong \operatorname{Hom}\left(R / \mathfrak{a},\left(0:_{H_{\mathfrak{a}}^{t}(M, N)} x\right)\right) \cong \operatorname{Hom}\left(R / \mathfrak{a}, H_{\mathfrak{a}}^{t}(M, N)\right)
$$

is in $S$.

\section{Asymptotic behaviour}

We keep the notations and hypotheses introduced in the introduction and we study the asymptotic stability of the associated primes of certain generalized local cohomology modules. The finiteness of graded generalized local cohomology module has an important role in studying the asymptotic behaviour of the $n$th graded component $H_{\mathfrak{a}}^{i}(M, N)_{n}$ of $H_{\mathfrak{a}}^{i}(M, N)$ for $n \rightarrow-\infty$. One of the parts of finiteness of graded generalized local cohomology is Artinianess. In the first part of this section, we prove that certain submodules of $H_{\mathfrak{a}}^{i}(M, N)$ are Artinian.

Definition 3.1. For an ideal $\mathfrak{a}$ in $R$, the finiteness dimension of $M$ and $N$ with respect to $\mathfrak{a}$, denoted $f_{\mathfrak{a}}(M, N)$, is defined by

$$
f_{\mathfrak{a}}(M, N)=\inf \left\{i \in \mathbb{N}_{0} \mid H_{\mathfrak{a}}^{i}(M, N) \text { is not finitely generated }\right\} .
$$


Also, for graded ideal $\mathfrak{a}$ in $R$, the generalized homological finite length dimension of $N$ and $M$ with respect to $\mathfrak{a}$ is defined as

$g_{\mathfrak{a}}(M, N)=\inf \left\{i \in \mathbb{N}_{0} \mid l_{R_{0}} H_{\mathfrak{a}}^{i}(M, N)_{n}=\infty\right.$ for infinitely many $\left.n \in \mathbb{Z}\right\}$.

In addition, the notation $\operatorname{cd}_{\mathfrak{a}}(M, N)$ is the largest non-negative integer $i$ such that $H_{\mathfrak{a}}^{i}(M, N)$ is not equal to zero. In particular $\operatorname{cd}_{\mathfrak{a}}(R, N)=\operatorname{cd}_{\mathfrak{a}}(N)$.

At this stage the following remark is needed.

Remark 3.2. Let $R=\bigoplus_{n \geq 0} R_{n}$. Then the following hold:

(i) If $T=\bigoplus_{n \in \mathbb{Z}} T_{n}$ is a finitely generated graded $R$-module, then $T_{n}=0$ for all $n \ll 0$.

(ii) If $T=\bigoplus_{n \in \mathbb{Z}} T_{n}$ is a Noetherian (resp. Artinian) graded $R$-module, then $T_{n}$ is Noetherian (resp. Artinian) $R_{0}$-module for all $n \in \mathbb{Z}$.

Theorem 3.3. Let $\mathfrak{b}_{0}$ be an ideal of $R_{0}$ such that $\mathfrak{a}_{0}+\mathfrak{b}_{0}$ is an $\mathfrak{m}_{0}$-primary ideal, $M$ and $N$ finitely generated graded $R$-modules. Let $i \leq f_{\mathfrak{a}}(M, N)$. Then $H_{\mathfrak{b}_{0} R}^{j}\left(H_{\mathfrak{a}}^{i}(M, N)\right)$ is Artinian for $j=0,1$.

Proof. If $i<f_{\mathfrak{a}}(M, N)$, then, in view of the definition of $f_{\mathfrak{a}}(M, N)$ and Remark 3.2, $H_{\mathfrak{b}_{0} R}^{j}\left(H_{\mathfrak{a}}^{i}(M, N)\right)$ is a direct sum of finitely many $R_{0}$-modules $H_{\mathfrak{b}_{\mathfrak{o}}+\mathfrak{a}_{\mathfrak{o}}}^{j}\left(H_{\mathfrak{a}}^{i}(M, N)_{n}\right) \cong H_{\mathfrak{m}_{\mathfrak{o}}}^{j}\left(H_{\mathfrak{a}}^{i}(M, N)_{n}\right) \cong H_{\mathfrak{b}_{\mathfrak{o}}}^{j}\left(H_{\mathfrak{a}}^{i}(M, N)_{n}\right)$, so that it is an Artinian $R$-module. Thus we consider the case where $i=f_{\mathfrak{a}}(M, N)$. To this end, consider the Grothendieck's spectral sequence

$$
E_{2}^{p, q}=H_{\mathfrak{b}_{0} R}^{p}\left(H_{\mathfrak{a}}^{q}(M, N)\right) \Longrightarrow H_{\mathfrak{m}}^{p+q}(M, N) .
$$

Set $S=\{T \mid T$ is an Artinian $R$-module $\}$. It follows from previous paragraph that $H_{\mathfrak{b}_{\mathfrak{o}} R}^{p}\left(H_{\mathfrak{a}}^{q}(M, N)\right)$ is in $S$ for all $q<f_{\mathfrak{a}}(M, N)=f$. Note that $H_{\mathfrak{m}}^{i}(M, N)$ is $S$ for all $i \in \mathbb{N}_{0}$. By Using an argument similar to the proof of Theorem 2.2 , we obtain that $H_{\mathfrak{b}_{0} R}^{j}\left(H_{\mathfrak{a}}^{f}(M, N)\right)$ is in $S$ for $j=0,1$; and hence the result follows.

Corollary 3.4. Let $\left(R_{0}, \mathfrak{m}_{0}\right)$ be local and $M, N$ be two finitely generated and graded $R$-modules. Let $i \leq f_{\mathfrak{a}}(M, N)$. Then $H_{\mathfrak{m}_{0} R}^{j}\left(H_{\mathfrak{a}}^{i}(M, N)\right)$ is Artinian for $j=0,1$.

Proof. It follows immediately by using Grothendieck's spectral sequence

$$
E_{2}^{p, q}=H_{\mathfrak{m}_{0} R}^{p}\left(H_{\mathfrak{a}}^{q}(M, N)\right) \Longrightarrow H_{\mathfrak{m}}^{p+q}(M, N)
$$

and Theorem 3.3.

Lemma 3.5. Let $M$ and $N$ be two finitely generated graded $R$-modules. Let $\mathfrak{a}_{0}$ be an ideal of $R_{0}$ such that $\operatorname{dim} R_{0} / \mathfrak{a}_{0} \leq 1$. Then $H_{\mathfrak{m}_{0} R}^{j}\left(H_{\mathfrak{a}}^{i}(M, N)\right)$ is Artinian for all $i$ and $j=0,1$. 
Proof. If $\operatorname{dim} R_{0} / \mathfrak{a}_{0}=0$, then $R / \mathfrak{a}$ is Artinian. As a result of [2, Exercise 7.1.4], $H_{\mathfrak{a}}^{i}(N)$ is Artinian for all $i \in \mathbb{N}_{0}$. Set $S=\{T \mid T$ is an Artinian $R$-module $\}$. By Theorem 2.1, $H_{\mathfrak{a}}^{i}(M, N)$ is in $S$. Therefore, in view of the spectral sequence

$$
H_{\mathfrak{m}_{0} R}^{p}\left(H_{\mathfrak{a}}^{q}(M, N)\right) \Longrightarrow H_{\mathfrak{m}}^{p+q}(M, N),
$$

the result follows by similar argument as used in Theorem 2.2. So, we may assume that $\operatorname{dim} R_{0} / \mathfrak{a}_{0}=1$. Then there exists $x \in \mathfrak{m}_{0}$ which avoids all minimal primes of $\mathfrak{a}_{0}$; so that $\sqrt{\mathfrak{a}_{0}+x R_{0}}=\mathfrak{m}_{0}$. Since, by [14, 11.38], the Grothendieck's spectral sequence $E_{2}^{p, q}=H_{x R}^{p}\left(H_{\mathfrak{a}}^{q}(M, N)\right)$ converges to $H^{p+q}=H_{(\mathfrak{a}, x)}^{p+q}(M, N)$. It is clear $E_{2}^{p, q}=0$ unless $p=0,1$, it follows that the exact sequence $0 \longrightarrow$ $E_{2}^{1, i-1} \longrightarrow H^{i} \longrightarrow E_{2}^{0, i} \longrightarrow 0$ which in turn yields the exact sequence

$$
0 \longrightarrow H_{x R}^{1}\left(H_{\mathfrak{a}}^{i-1}(M, N)\right) \longrightarrow H_{(\mathfrak{a}, x)}^{i}(M, N) \longrightarrow H_{x R}^{0}\left(H_{\mathfrak{a}}^{i}(M, N)\right) \longrightarrow 0 .
$$

As $\sqrt{(\mathfrak{a}, x)}=\mathfrak{m}_{0}+R_{+}=\mathfrak{m}$ is a maximal ideal of $R$, the $R$-module $H_{(\mathfrak{a}, x)}^{i}(M, N)$ is Artinian for all $i$. This proves the claim.

Theorem 3.6. Let $\left(R_{0}, \mathfrak{m}_{0}\right)$ be a local ring and $\mathfrak{b}_{0}$ an ideal of $R_{0}$ such that $\mathfrak{a}_{0}+\mathfrak{b}_{0}$ is an $\mathfrak{m}_{0}$-primary ideal. Let $N$ be a finitely generated graded $R$-module. Then $c d_{\mathfrak{a}}(N)=\operatorname{dim} N / \mathfrak{b}_{0} N=d$.

Proof. We argue by induction on $d$. It is straightforward to see that the result is true when $d=0$. Now, inductively assume that $d>0$ and that the assertion has been proved for $d-1$. It follows from $\left[2,2.1 .7(\right.$ iii) $]$ that $H_{\mathfrak{a}}^{i}(N)=H_{\mathfrak{a}}^{i}\left(N / \Gamma_{\mathfrak{a}}(N)\right)$ for all $i \geq 1$. Also, $N / \Gamma_{\mathfrak{a}}(N)$ is an $\mathfrak{a}$-torsion free $R$-module, by [2, 2.1.2]. Hence we can assume that $N$ is an $\mathfrak{a}$-torsion free $R$-module. Then the ideal $\mathfrak{a}$ contains an element $x$ which avoids all members of $\operatorname{Ass} N$ and $\min \operatorname{Ass}\left(N / \mathfrak{b}_{0} N\right)$. It is clear that $\operatorname{dim}(N / x N) / \mathfrak{b}_{0}(N / x N)=d-1$. Therefore, the exact sequence $0 \longrightarrow N \longrightarrow N \longrightarrow N / x N \longrightarrow 0$ induces a long exact sequence

$$
H_{\mathfrak{a}}^{d-1}(N / x N) \longrightarrow H_{\mathfrak{a}}^{d}(N) \longrightarrow H_{\mathfrak{a}}^{d}(N) \longrightarrow 0 .
$$

Now, let $i>d$, then $H_{\mathfrak{a}}^{i}(N) \cong x H_{\mathfrak{a}}^{i}(N)$. As $x \in \mathfrak{a}$ it follows $H_{\mathfrak{a}}^{i}(N)=0$. It is shown that $H_{\mathfrak{a}}^{d}(N) \neq 0$. By using the exact sequence $0 \longrightarrow \mathfrak{b}_{0} N \longrightarrow$ $N \longrightarrow N / \mathfrak{b}_{0} N \longrightarrow 0$, we get an exact sequence $H_{\mathfrak{a}}^{i}\left(\mathfrak{b}_{0} N\right) \longrightarrow H_{\mathfrak{a}}^{i}(N) \longrightarrow$ $H_{\mathfrak{a}}^{i}\left(N / \mathfrak{b}_{0} N\right)$. Also, $H_{\mathfrak{a}}^{i}\left(\mathfrak{b}_{0} N\right)=0$ for all $i>d$ and $H_{\mathfrak{a}}^{i}(N) \cong H_{\mathfrak{a}}^{i}\left(N / \mathfrak{b}_{0} N\right)$, because $\operatorname{dim}\left(\mathfrak{b}_{0} N / \mathfrak{b}_{0}\left(\mathfrak{b}_{0} N\right)\right) \leq d$. As $\sqrt{\mathfrak{a}+\mathfrak{b}_{0}}=\mathfrak{m}$ is a maximal ideal, the $R$ module $H_{\mathfrak{a}}^{i}\left(N / \mathfrak{b}_{0} N\right) \cong H_{\mathfrak{a}+\mathfrak{b}_{0}}^{i}\left(N / \mathfrak{b}_{0} N\right) \cong H_{\mathfrak{m}}^{i}\left(N / \mathfrak{b}_{0} N\right) \neq 0$, it follows $H_{\mathfrak{a}}^{d}(N) \neq$ 0 , see $[2,6.1 .4]$.

Theorem 3.6 yields the following.

Corollary 3.7. Assume that $\left(R_{0}, \mathfrak{m}_{0}\right)$ is local, $\mathfrak{b}_{0}$ an ideal of $R_{0}$ such that $\mathfrak{a}_{0}+\mathfrak{b}_{0}$ is an $\mathfrak{m}_{0}$-primary ideal. Let $M$ be a finitely generated $R$-module with $p d(M)=\ell$ and $N$ a finitely generated graded $R$-module. If $\operatorname{dim} N / \mathfrak{b}_{0} N=d$, then $c d_{\mathfrak{a}}(M, N) \leq d+\ell$. 
Proof. We argue by induction on $\ell$. If $\ell=0$, then $M$ is a finitely generated free $R$-module, and the assertion is trivial, by Theorem 3.6. Let $\ell>0$ and the result has been proved for $\ell-1$. Now, we consider the exact sequence $0 \longrightarrow M^{\prime} \longrightarrow R^{n} \longrightarrow M \longrightarrow 0$, to obtain the exact sequence $H_{\mathrm{a}}^{i-1}\left(M^{\prime}, N\right) \longrightarrow$ $H_{\mathfrak{a}}^{i}(M, N) \longrightarrow H_{\mathfrak{a}}^{i}\left(R^{n}, N\right) \longrightarrow H_{\mathfrak{a}}^{i}\left(M^{\prime}, N\right)$. Then the result follows from the above exact sequence and inductive hypothesis.

In the remaining part of this section, we study the asymptotic behaviour on generalized local cohomology modules. We consider the following statements.

(i) $\operatorname{Ass}_{R_{0}} H_{\mathfrak{a}}^{i}(M, N)_{n}$ is asymptotically stable for $n \rightarrow-\infty$, if there is some $n_{0} \in \mathbb{Z}$ such that $\operatorname{Ass}_{R_{0}} H_{\mathfrak{a}}^{i}(M, N)_{n}=\operatorname{Ass}_{R_{0}} H_{\mathfrak{a}}^{i}(M, N)_{n_{0}}$ for all $n \leq n_{0}$,

(ii) $H_{\mathfrak{a}}^{i}(M, N)$ is tame or asymptotically gap free if there is some $n_{0} \in \mathbb{Z}$ such that $H_{\mathfrak{a}}^{i}(M, N)_{n}=0$ for all $n \leq n_{0}$ or else $H_{\mathfrak{a}}^{i}(M, N)_{n} \neq 0$ for all $n \leq n_{0}$.

In the next lemma we deal with cofiniteness property of generalized local cohomology modules. Recall that an $R$-module $T$ is said to be $\mathfrak{a}$-cofinite if $\operatorname{Supp} T \subseteq V(\mathfrak{a})$ and either $\operatorname{Ext}_{R}^{i}(R / \mathfrak{a}, T)$ is finite for all $i$.

Lemma 3.8. Suppose that $\left(R_{0}, \mathfrak{m}_{0}\right)$ is local, $\mathfrak{b}_{0}$ an ideal of $R_{0}$ such that $\mathfrak{a}_{0}+\mathfrak{b}_{0}$ is an $\mathfrak{m}_{0}$-primary ideal. Let $M$ be a finitely generated and graded $R$-module with $p d(M)=\ell$. If $N$ is a finitely generated and graded $R$-module such that $\operatorname{dim} N / \mathfrak{b}_{0} N=d$, then $H_{\mathfrak{a}}^{d+\ell}(M, N) / \mathfrak{b}_{0} H_{\mathfrak{a}}^{d+\ell}(M, N)$ is Artinian and $\mathfrak{a}$-cofinite. Moreover $H_{\mathfrak{a}}^{d+\ell}(M, N)$ is tame.

Proof. We prove this by induction on $\operatorname{dim} N / \mathfrak{b}_{0} N=d$. If $d=0$, then $\Gamma_{\mathfrak{a}}(N)=$ $N$ and hence $H_{\mathfrak{a}}^{d+\ell}(M, N) \cong \operatorname{Ext}_{R}^{d+\ell}(M, N)$. Therefore, since the radical of the annihilator of $H_{\mathfrak{a}}^{d+\ell}(M, N) / \mathfrak{b}_{0} H_{\mathfrak{a}}^{d+\ell}(M, N)$ is equal to $\mathfrak{m}$, the $R$-module $H_{\mathfrak{a}}^{d+\ell}(M, N) / b_{0} H_{\mathfrak{a}}^{d+\ell}(M, N)$ is Artinian and a-cofinite. Suppose, inductively, that $d>0$ and the result has been proved for $d-1$. Now, the exact sequence $0 \longrightarrow \Gamma_{\mathfrak{a}}(N) \longrightarrow N \longrightarrow N / \Gamma_{\mathfrak{a}}(N) \longrightarrow 0$ is used, in conjunction with the facts that $H_{\mathfrak{a}}^{i}\left(M, \Gamma_{\mathfrak{a}}(N)\right) \cong \operatorname{Ext}_{R}^{i}\left(M, \Gamma_{\mathfrak{a}}(N)\right)$ and $\operatorname{pd}(M)=\ell$, to see that $H_{\mathfrak{a}}^{d+\ell}(M, N) \cong H_{\mathfrak{a}}^{d+\ell}\left(M, N / \Gamma_{\mathfrak{a}}(N)\right)$. Therefore, we may assume that $N$ is $\mathfrak{a}$-torsion-free. Hence there exists a homogeneous element $x$ which avoids all members of $\operatorname{Ass} N$ and $\min \operatorname{Ass}\left(N / \mathfrak{b}_{\mathcal{o}} N\right)$. It is straightforward to see that $\operatorname{dim}(N / x N) / \mathfrak{m}_{\mathfrak{o}}(N / x N)=d-1$. Thus, $H_{\mathfrak{a}}^{d+\ell}(M, N / x N)=0$, by Corollary 3.7. Therefore, the exact sequence

$$
0 \longrightarrow N \stackrel{x}{\longrightarrow} N \longrightarrow N / x N \longrightarrow 0
$$

induces the exact sequence

$$
H_{\mathfrak{a}}^{d+\ell-1}(M, N / x N) \longrightarrow H_{\mathfrak{a}}^{d+\ell}(M, N) \stackrel{x}{\longrightarrow} H_{\mathfrak{a}}^{d+\ell}(M, N) \longrightarrow 0,
$$

which in turn yields the exact sequence

$$
\begin{aligned}
& H_{\mathfrak{a}}^{d+\ell-1}(M, N / x N) / \mathfrak{b}_{\mathfrak{o}} H_{\mathfrak{a}}^{d+\ell-1}(M, N / x N) \longrightarrow \\
& \quad H_{\mathfrak{a}}^{d+\ell}(M, N) / \mathfrak{b}_{\mathcal{o}} H_{\mathfrak{a}}^{d+\ell}(M, N) \stackrel{x}{\longrightarrow} H_{\mathfrak{a}}^{d+\ell}(M, N) / \mathfrak{b}_{\mathfrak{o}} H_{\mathfrak{a}}^{d+\ell}(M, N) \longrightarrow 0
\end{aligned}
$$


By using the above exact sequence in conjunction with the inductive hypothesis

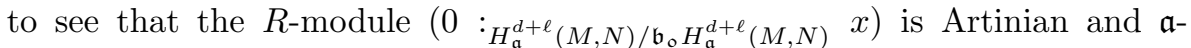
cofinite. Therefore, in view of $[13,4.1], H_{\mathfrak{a}}^{d+\ell}(M, N) / \mathfrak{b}_{0} H_{\mathfrak{a}}^{d+\ell}(M, N)$ is Artinian and a-cofinite. In particular, the $R$-module $H_{\mathfrak{a}}^{d+\ell}(M, N) / \mathfrak{b}_{0} H_{\mathfrak{a}}^{d+\ell}(M, N)$ is tame. It therefore follows, in view of Nakayama's Lemma, that $H_{\mathfrak{a}}^{d+\ell}(M, N)$ is tame.

The following lemma is needed in the proof of the next theorem.

Lemma 3.9. Let $M, N$ be finitely generated graded $R$-modules. Then the following statements are proved:

(i) If $j<t$ and $H_{\mathfrak{a}}^{j}(M, N)_{n}$ is a finitely generated $R_{0}$-module for all $n \ll 0$, then $\operatorname{Ass}_{R_{0}} H_{\mathfrak{a}}^{j}(M, N)_{n}$ is a finite set for all $j \leq t$ and $n \ll 0$,

(ii) Let $\Gamma_{\mathfrak{a}_{0}}(M)=M$ and $n_{0} \in \mathbb{Z}$ be such that, for all $j<t, H_{\mathfrak{a}}^{j}(M, N)_{n}=$ 0 for all $n \leqslant n_{0}$. Then, $\operatorname{Ass}_{R_{0}} H_{\mathfrak{a}}^{j}(M, N)_{n}$ is asymptotically stable as $n \rightarrow-\infty$ for all $j \leq t$,

(iii) If $i \leq g_{\mathfrak{a}}(M, N)$ and $\operatorname{Ass}_{R_{0}} H_{\mathfrak{a}}^{i}(M, N)_{n}-\left\{\mathfrak{m}_{0}\right\}$ is asymptotically stable as $n \rightarrow-\infty$, then $\operatorname{Ass}_{R_{0}} H_{\mathfrak{a}}^{i}(M, N)_{n}$ is asymptotically stable as $n \rightarrow$ $-\infty$ for all $i \leq g_{\mathfrak{a}}(M, N)$.

Proof. (i) Set $S=\left\{T=\bigoplus_{n \in \mathbb{Z}} T_{n} \mid T_{n}\right.$ is a finitely generated $R_{0}$-module for all $n \ll 0\}$. By the hypotheses $\operatorname{Ass}_{R_{0}} H_{\mathfrak{a}}^{i}(M, N)_{n}$ is a finite set for all $i<t$ and $n \ll 0$. Therefore, it is enough to show that $\operatorname{Hom}\left(R / \mathfrak{a}, H_{\mathfrak{a}}^{t}(M, N)\right)$ is in $S$. To do this, we apply induction on $t$. If $t=0$, then $\operatorname{Hom}\left(R / \mathfrak{a}, H_{\mathfrak{a}}^{0}(M, N)\right) \cong$ $\operatorname{Hom}\left(R / \mathfrak{a}, \operatorname{Hom}\left(M, \Gamma_{\mathfrak{a}}(N)\right) \cong \operatorname{Hom}\left(M / \mathfrak{a} M, \Gamma_{\mathfrak{a}}(N)\right)\right.$ is in $S$. Suppose that $t>0$, and the result holds for the case $t-1$. Since, $H_{\mathfrak{a}}^{i}\left(M, \Gamma_{\mathfrak{a}}(N)\right) \cong$ $\operatorname{Ext}_{R}^{i}\left(M, N / \Gamma_{\mathfrak{a}}(N)\right)$ for all $i$, it follows from Remark 3.2, that

$$
\operatorname{Hom}\left(R / \mathfrak{a}, H_{\mathfrak{a}}^{t}(M, N)\right.
$$

is in $S$ if and only if $\operatorname{Hom}\left(R / \mathfrak{a}, H_{\mathfrak{a}}^{t}\left(M, N / \Gamma_{\mathfrak{a}}(N)\right)\right)$ is in $S$. Thus we may assume that $\Gamma_{\mathfrak{a}}(N)=0$. Then there is an element $x \in \mathfrak{a}$ which is a non-zerodivisor on $N$. The exact sequence $0 \longrightarrow N \stackrel{x}{\longrightarrow} N \longrightarrow N / x N \longrightarrow 0$ implies the following long exact sequence of generalized local cohomology modules

$$
H_{\mathfrak{a}}^{t-1}(M, N) \stackrel{\lambda}{\longrightarrow} H_{\mathfrak{a}}^{t-1}(M, N / x N) \stackrel{\varphi}{\longrightarrow} H_{\mathfrak{a}}^{t}(M, N) \stackrel{x}{\longrightarrow} H_{\mathfrak{a}}^{t}(M, N) .
$$

The $R$-module $\operatorname{Hom}\left(R / \mathfrak{a}, H_{\mathfrak{a}}^{t-1}(M, N / x N)\right)$ is in $S$, by using the exact sequence in conjunction with the inductive hypothesis. By $[6,2.1], \operatorname{Ext}_{R}^{1}(R / \mathfrak{a}, \operatorname{im} \lambda)$ is in $S$. Therefore, $\operatorname{Hom}(R / \mathfrak{a}, \operatorname{im} \varphi) \cong \operatorname{Hom}\left(R / \mathfrak{a},\left(0:_{H_{\mathfrak{a}}^{t}(M, N)} x\right)\right)$ is in $S$. The following completes the proof:

$$
\begin{aligned}
\operatorname{Hom}\left(R / \mathfrak{a}, H_{\mathfrak{a}}^{t}(M, N)\right) & \cong \operatorname{Hom}\left(R / \mathfrak{a} \bigotimes R / x R, H_{\mathfrak{a}}^{t}(M, N)\right. \\
& \cong \operatorname{Hom}\left(R / \mathfrak{a},\left(0:_{H_{\mathfrak{a}}^{t}(M, N)} x\right)\right) .
\end{aligned}
$$

(ii) We prove by induction on $t$. The result is clear when $t=0$. So, let $0<$ $t$ and assume that the result has been proved for smaller values of $t$. The 
hypotheses give $H_{\mathfrak{a}}^{i}\left(M, \Gamma_{R_{+}}(N)\right) \cong \operatorname{Ext}_{R}^{i}\left(M, \Gamma_{R_{+}}(N)\right)$. Now, we can use the graded exact sequence

$$
H_{\mathfrak{a}}^{t}\left(M, \Gamma_{R_{+}}(N)\right) \longrightarrow H_{\mathfrak{a}}^{t}(M, N) \longrightarrow H_{\mathfrak{a}}^{t}\left(M, N / \Gamma_{R_{+}}(N)\right) \longrightarrow H_{\mathfrak{a}}^{t+1}\left(M, \Gamma_{R_{+}} N\right)
$$

to see that $H_{\mathfrak{a}}^{t}(M, N)_{n} \cong H_{\mathfrak{a}}^{t}\left(M, N / \Gamma_{R_{+}}(N)\right)_{n}$ for all $n \ll 0$. We may assume that $\Gamma_{R_{+}}(N)=0$. Using prime avoidance theorem, we can get a homogeneous $N$-regular element $x$ of positive degree $k$. The exact sequence $0 \longrightarrow N \longrightarrow$ $N \longrightarrow N / x N \longrightarrow 0$ induces the long exact sequence of $R_{0}$-module

$$
H_{\mathfrak{a}}^{t-1}(M, N)_{n} \longrightarrow H_{\mathfrak{a}}^{t-1}(M, N)_{n+k} \longrightarrow H_{\mathfrak{a}}^{t-1}(M, N / x N)_{n+k} \longrightarrow H_{\mathfrak{a}}^{t}(M, N)_{n}
$$

which shows that $H_{\mathfrak{a}}^{i}(M, N / x N)_{n}=0$ for all $i<t-1$ and $n \ll 0$. Therefore, there is the exact sequence

$$
0 \longrightarrow H_{\mathfrak{a}}^{t-1}(M, N / x N)_{n+k} \longrightarrow H_{\mathfrak{a}}^{t}(M, N)_{n} \longrightarrow H_{\mathfrak{a}}^{t}(M, N)_{n+k}
$$

which, in turn, yields

$$
\begin{aligned}
& \operatorname{Ass}_{R_{0}} H_{\mathfrak{a}}^{t-1}(M, N / x N)_{n+k} \\
\subseteq & \operatorname{Ass}_{R_{0}} H_{\mathfrak{a}}^{t}(M, N)_{n} \\
\subseteq & \operatorname{Ass}_{R_{0}} H_{\mathfrak{a}}^{t-1}(M, N / x N)_{n+k} \bigcup \operatorname{Ass}_{R_{0}} H_{\mathfrak{a}}^{t}(M, N)_{n+k} .
\end{aligned}
$$

The inductive hypothesis and $(*)$ show that

$$
\operatorname{Ass}_{R} H_{\mathfrak{a}}^{t}(M, N)_{n} \subseteq \operatorname{Ass}_{R} H_{\mathfrak{a}}^{t}(M, N)_{n+k} \text { for all } n \ll 0 .
$$

By (i), the set $\operatorname{Ass}_{R_{0}} H_{\mathfrak{a}}^{t}(M, N)_{n}$ is finite for all $n \ll 0$, this completes the inductive step and the proof.

(iii) Let $\mathfrak{m}=\mathfrak{m}_{0}+R_{+}$and consider the Grothendieck's spectral sequence

$$
\left(E_{2}^{p, i}\right)_{n}=H_{\mathfrak{m}_{0} R}^{p}\left(H_{\mathfrak{a}}^{i}(M, N)\right)_{n} \Longrightarrow H_{\mathfrak{m}}^{p+i}(M, N)_{n} .
$$

If $i<g_{\mathfrak{a}}(M, N)$, then, in view of the definition of $g_{\mathfrak{a}}(M, N), l_{R_{0}} H_{\mathfrak{a}}^{i}(M, N)_{n}$ is finite for all $n \ll 0$ and the result is clear. In addition, it follows that there exists $n_{0} \in \mathbb{Z}$ such that for all $n \leq n_{0},\left(E_{2}^{p, i}\right)_{n}=0$ for $i<g_{\mathfrak{a}}(M, N)$ and $p \in \mathbb{N}$. Now, the convergence of the above spectral sequence implies that $H_{\mathfrak{m}_{0} R}^{0}\left(H_{\mathfrak{a}}^{g}(M, N)\right)_{n} \cong H_{\mathfrak{m}}^{g}(M, N)_{n}$ for all $n \leq n_{0}$. Since all graded Artinian $R$-modules are tame, it is seen $H_{\mathfrak{m}_{0} R}^{0}\left(H_{\mathfrak{a}}^{g}(M, N)\right)$ is tame and

$$
\operatorname{Supp}_{R_{0}} \Gamma_{\mathfrak{m}_{0}}\left(H_{\mathfrak{a}}^{g}(M, N)_{n}\right) \subseteq\left\{\mathfrak{m}_{0}\right\}
$$

for all $n \ll 0$. Now, the result follows using the exact sequence

$$
\begin{aligned}
0 \longrightarrow \Gamma_{\mathfrak{m}_{0}}\left(H_{\mathfrak{a}}^{g}(M, N)_{n}\right) & \longrightarrow H_{\mathfrak{a}}^{g}(M, N)_{n} \\
& \longrightarrow H_{\mathfrak{a}}^{g}(M, N)_{n} / \Gamma_{\mathfrak{m}_{0}}\left(H_{\mathfrak{a}}^{g}(M, N)_{n}\right) \longrightarrow 0
\end{aligned}
$$

Theorem 3.10. Let $M$ and $N$ be finitely generated graded $R$-modules. Let $\Gamma_{\mathfrak{a}_{0}}(M)=M$ and $i \leq g_{\mathfrak{a}}(M, N)$. Then the set $\operatorname{Ass}_{R_{0}} H_{\mathfrak{a}}^{i}(M, N)_{n}$ is asymptotically stable as $n \rightarrow-\infty$. 
Proof. It is enough, in view Lemma 3.9(iii), to show the set $\operatorname{Ass}_{R_{0}} H_{\mathfrak{a}}^{i}(M, N)_{n}-$ $\left\{\mathfrak{m}_{0}\right\}$ is asymptotically stable for all $i \leq g_{\mathfrak{a}}(M, N)=g$. Using the concept of $g_{\mathfrak{a}}(M, N)$, it is easy to see that the result is clear, for all $i<g_{\mathfrak{a}}(M, N)$. Thus we consider the case where $i=g_{\mathfrak{a}}(M, N)$. Let $\left(\hat{R}_{0}, \hat{\mathfrak{m}}_{0}\right)$ denote the $\mathfrak{m}_{0}$-adic completion of the local ring $\left(R_{0}, \mathfrak{m}_{0}\right)$ and let $M \bigotimes_{R_{0}} \hat{R}_{0}=\hat{M}$ and $N \bigotimes_{R_{0}} \hat{R}_{0}=$ $\hat{N}$. In view of $[5,3.1]$ we may assume that $R_{0}$ is complete. Then the set $A=\bigcup_{n \ll 0} \operatorname{Ass}_{R_{0}} H_{\mathfrak{a}}^{g}(M, N)_{n}-\left\{\mathfrak{m}_{0}\right\}$ is not empty. By [11, 3.2], there exists $x \in \mathfrak{m}_{0}$ such that $x \notin \bigcup_{p \in A} p$. Let $S=\left\{x^{t} \mid 0 \leq t \in \mathbb{Z}\right\}$. Then $S \cap \mathfrak{m}_{0} \neq \varnothing$. If $i<$ $g_{\mathfrak{a}}(M, N)$, then $l_{R_{0}} H_{\mathfrak{a}}^{i}(M, N)_{n}<\infty$ for all $n \ll 0$. Hence $\left(H_{\mathfrak{a} R_{x}}^{i}\left(M_{x}, N_{x}\right)\right)_{n}=0$ for all $n \ll 0$. Therefore, by Lemma 3.9(ii) the set $\operatorname{Ass}_{\left(R_{0}\right)_{x}} H_{\mathfrak{a} R_{x}}^{i}\left(M_{x}, N_{x}\right)_{n}$ is asymptotically stable for all $i \leq g$, when $n \rightarrow-\infty$. Hence there exists $n_{0} \in \mathbb{Z}$ such that

$$
\operatorname{Ass}_{\left(R_{0}\right)_{x}} H_{\mathfrak{a} R_{x}}^{g}\left(M_{x}, N_{x}\right)_{n}=\operatorname{Ass}_{\left(R_{0}\right)_{x}} H_{\mathfrak{a} R_{x}}^{g}\left(M_{x}, N_{x}\right)_{n_{0}}
$$

for all $n \leq n_{0}$. Thus $\operatorname{Ass}_{R_{0}} H_{\mathfrak{a}}^{g}(M, N)_{n}-\left\{\mathfrak{m}_{0}\right\}=\operatorname{Ass}_{R_{0}} H_{\mathfrak{a}}^{g}(M, N)_{n_{0}}-\left\{\mathfrak{m}_{0}\right\}$ for all $n \leq n_{0}$. This completes the proof.

Proposition 3.11. Let $M$ and $N$ be finitely generated graded $R$-modules. Let $\mathfrak{a}_{\mathfrak{o}}$ be nilpotent and $i \leq f_{\mathfrak{a}}(M, N)$. Then the set $\operatorname{Ass}_{R_{0}} H_{\mathfrak{a}}^{i}(M, N)_{n}$ is asymptotically stable as $n \rightarrow-\infty$.

Proof. It is proved by induction on $f_{\mathfrak{a}}(M, N)=f$. It is easy to see

$$
H_{\mathfrak{a}}^{0}(M, N)_{n} \cong \operatorname{Hom}\left(M, \Gamma_{\mathfrak{a}}(N)\right)_{n}=0
$$

for all $n \ll 0$. It follows that $\operatorname{Ass}_{R_{0}} H_{\mathfrak{a}}^{0}(M, N)_{n}$ is empty for all $n \ll 0$. So, let $f>0$ and assume that the result has been proved for smaller values of $f$. The hypotheses lead to $H_{\mathfrak{a}}^{i}\left(M, \Gamma_{R_{+}}(N)\right) \cong \operatorname{Ext}_{R}^{i}\left(M, \Gamma_{R_{+}}(N)\right)$ for all $i \geq 0$. We can use the graded exact sequence $H_{\mathfrak{a}}^{f}\left(M, \Gamma_{R_{+}}(N)\right) \longrightarrow H_{\mathfrak{a}}^{f}(M, N) \longrightarrow$ $H_{\mathfrak{a}}^{f}\left(M, N / \Gamma_{R_{+}}(N)\right)$, to see that

$$
H_{\mathfrak{a}}^{f}(M, N)_{n} \cong H_{\mathfrak{a}}^{f}\left(M, N / \Gamma_{R_{+}}(N)\right)_{n}
$$

for all $n \ll 0$. Hence, by replacing $N$ with $N / \Gamma_{R_{+}}(N)$, we may assume that there exists a homogeneous element $x \in R_{+}$, say of degree $k$, which is a nonzerodivisor on $N$. The application of generalized local cohomology with respect to $\mathfrak{a}$ to exact sequence $0 \longrightarrow N \stackrel{x}{\longrightarrow} N \longrightarrow N / x N \longrightarrow 0$ leads to an exact sequence of $R_{0}$-modules

$$
H_{\mathfrak{a}}^{f-1}(M, N) \longrightarrow H_{\mathfrak{a}}^{f-1}(M, N / x N) \longrightarrow H_{\mathfrak{a}}^{f}(M, N) \stackrel{x}{\longrightarrow} H_{\mathfrak{a}}^{f}(M, N) .
$$

The sequence first show that $H_{\mathfrak{a}}^{i}(M, N / x N)$ is finitely generated for all $i<f$. So, by inductive hypothesis, there is some $n_{0} \in \mathbb{Z}$ such that

$$
B=\operatorname{Ass}_{R_{0}} H_{\mathfrak{a}}^{f-1}(M, N / x N)_{n}=\operatorname{Ass}_{R_{0}} H_{\mathfrak{a}}^{f-1}(M, N / x N)_{n_{0}}
$$

for all $n \leq n_{0}$. Moreover, there is some integer $n_{1}<n_{0}$ such that

$$
H_{\mathfrak{a}}^{f-1}(M, N)_{n+k}=0
$$


for all $n \leq n_{1}$ by Remark 3.2. So, for all $n \leq n_{1}$, we have an exact sequence of $R_{0}$-modules

$$
0 \longrightarrow H_{\mathfrak{a}}^{f-1}(M, N / x N)_{n+k} \longrightarrow H_{\mathfrak{a}}^{f}(M, N)_{n} \stackrel{x}{\longrightarrow} H_{\mathfrak{a}}^{f}(M, N)_{n+k},
$$

which, in turn, yields

$$
B \subseteq \operatorname{Ass}_{R_{0}} H_{\mathfrak{a}}^{f}(M, N)_{n} \subseteq B \cup \operatorname{Ass}_{R_{0}} H_{\mathfrak{a}}^{f}(M, N)_{n+k}
$$

for all $n \leq n_{1}$. This shows that $\operatorname{Ass}_{R_{0}} H_{\mathfrak{a}}^{f}(M, N)_{n} \subseteq \operatorname{Ass}_{R_{0}} H_{\mathfrak{a}}^{f}(M, N)_{n+k}$ for all $n \leq n_{1}$. Since, by Lemma 3.9(i), the set $\operatorname{Ass}_{R_{0}} H_{\mathfrak{a}}^{f}(M, N)_{n+k}$ is finite for all $n \ll 0$, and hence the result follows.

Notation. Let $i \in \mathbb{N}_{0}$. We set: $A_{n}^{i}=\left\{p_{0} \in \operatorname{Ass}_{R_{0}} H_{\mathfrak{a}}^{i}(M, N)_{n} \mid \operatorname{dim} R_{0} / p_{0} \geq\right.$ $1\}$.

Remark 3.12. $H_{\mathfrak{a}}^{i}(M, N)$ is an $R_{+}$-torsion module for all $i \in \mathbb{Z}$. So, we have $\operatorname{Ass}_{R} H_{\mathfrak{a}}^{i}(M, N) \subseteq V\left(R_{+}\right)$which yields the natural bijection

$$
\operatorname{Ass}_{R} H_{\mathfrak{a}}^{i}(M, N) \longrightarrow \bigcup_{n \in \mathbb{Z}} \operatorname{Ass}_{R_{0}}\left(H_{\mathfrak{a}}^{i}(M, N)_{n}\right) \quad\left(p \longmapsto p \cap R_{0}\right) .
$$

Theorem 3.13. Suppose that $M$ and $N$ are finitely generated $R$-modules and that $\operatorname{dim}\left(R_{0} / \mathfrak{a}_{0}\right)=1$. Then for all $i$ the set $\operatorname{Ass}_{R_{0}} H_{\mathfrak{a}}^{i}(M, N)_{n}$ is asymptotically stable, for $n \rightarrow-\infty$.

Proof. Using Lemma 3.5, $\Gamma_{m_{0} R} H_{\mathfrak{a}}^{i}(M, N)$ is tame. So, that either

$$
\mathfrak{m}_{0} \in \operatorname{Ass}_{R_{0}} H_{\mathfrak{a}}^{i}(M, N)_{n}
$$

for all $n \ll 0$ or else $\mathfrak{m}_{0} \notin \operatorname{Ass}_{R_{0}} H_{\mathfrak{a}}^{i}(M, N)_{n}$ for all $n \ll 0$. Therefore, it is enough to show that $A_{n}^{i}$ is asymptotically stable as, $n \rightarrow-\infty$. Since, in view of $[4,3.3], H_{\mathfrak{a}}^{i}(M, N)$ is $\mathfrak{a}$-cofinite for all $i \in \mathbb{N}_{0}$, by using $[12,1.4] \operatorname{Ass}_{R} H_{\mathfrak{a}}^{i}(M, N)$ is a finite set. Thus, by Remark 3.12 , it is deduced that $A_{n}^{i}$ and $\bigcup_{n \in \mathbb{N}} A_{n}^{i}$ are finite sets. Now, Let $p_{0} \in A_{n}^{i}$, then $\left(R_{0}\right)_{p_{0}}$ is a local ring of dimension 0 , and hence, $p_{0}\left(R_{0}\right)_{p_{0}} \in \operatorname{Ass}_{\left(R_{0}\right)_{p_{0}}}\left(H_{\mathfrak{a}}^{i}(M, N)_{p_{0}}\right)_{n}$ for all $n \ll 0$ or $p_{0}\left(R_{0}\right)_{p_{0}} \notin$ $\operatorname{Ass}_{\left(R_{0}\right) p_{0}}\left(H_{\mathfrak{a}}^{i}(M, N)_{p_{0}}\right)_{n}$ for all $n \ll 0$. In view of the natural isomorphism of $\left(R_{0}\right)_{p_{0}}$-module $\left(H_{\mathfrak{a}}^{i}(M, N)_{n}\right) p_{0} \cong H_{\mathfrak{a} p_{0}}^{i}\left(M_{p_{0}}, N_{p_{0}}\right)_{n}$, we thus see that either $p_{0} \in \operatorname{Ass}_{R_{0}} H_{\mathfrak{a}}^{i}(M, N)_{n}$ for all $n \ll 0$ or $p_{0} \notin \operatorname{Ass}_{R_{0}} H_{\mathfrak{a}}^{i}(M, N)_{n}$ for all $n \ll 0$. This proves the claim.

Acknowledgment. The author is deeply grateful to the referees for their careful reading and comments in the improvement of this paper.

\section{References}

[1] M. H. Bijan-Zadeh, A common generalization of local cohomology theories, Glasgow Math. J. 21 (1980), no. 2, 173-181.

[2] M. P. Brodmann and R. Y. Sharp, Local Cohomology: An Algebraic Introduction with Geometric Applications, Cambridge Univ. Press 1998.

[3] W. Bruns and J. Herzog, Cohen Macaulay Rings, Cambridge Univ. Press 1993 
[4] F. Dehghani-Zadeh, Cofinite modules and asymptotic behaviour of generalized local cohomology, Proceedings of the 5-th Asian Mathematical Conference, Malaysia 2009, 592602 .

[5] - On the finiteness properties of generalized local cohomology modules, Int. Electron. J. Algebra 10 (2011), 113-122.

[6] F. Dehghani-Zadeh and H. Zakeri, Some results on graded generalized local cohomology modules, J. Math. Ext. 5 (2010), no. 1, 59-73.

[7] J. Herzog, Komplexe, Auflösungen und Dualität in der Lokalen Algebra, Habilitationsschrift, Universität Regensburg, 1974.

[8] M. Jahangiri, N. Shirmohammadi, and Sh. Tahamtan, Tameness and Artinianness of graded generalized local cohomology modules, preprint.

[9] M. Jahangiri and H. Zakeri, Local cohomology modules with respect to an ideal containing the irrelevant ideal, J. Pure Appl. Algebra 213 (2009), no. 4, 573-581.

[10] T. Marley and J. Vassilev, Cofiniteness and associated primes of local cohomology modules, J. Algebra 256 (2002), no. 1, 180-193.

[11] H. Matsumura, Commutative Ring Theory, Cambridge Univ. Press 1986.

[12] L. Melkersson, Properties of cofinite modules and applications to local cohomology, Math. Proc. Cambridge Philos. Soc. 125 (1999), no. 3, 417-423.

[13] — Modules cofinite with respect to an ideal, J. Algebra 285 (2005), no. 2, 649-668.

[14] J. Rotman, An Introduction to Homological Algebra, Academic Press, Orlando, 1979.

Department of Mathematics

ISLAMIC AZAD UNIVERSITY

YAZD BRANCH

YAZD, IRAN

E-mail address: f.dehghanizadeh@yahoo.com; fdzadeh@gmail.com 